\title{
Keywords, Volume 9, 2004
}

\section{Achievement}

Achievement goals

Action organization

Adolescents

Alphabetic

American

Attachment

Attachment styles

Attention

BCS70

Burnout

Central executive

Child-rearing attitudes

Classroom

Cognitive strategies

Computer supported learning

Conceptualizations

Congresses

Context

Cross-linguistic

Detox

Disorganization

Drug abuse

Dual-task

Dynamic of writing

Educational performance

Egocentrism fallacy

Emotion

Empathy

Ethics

Euro diploma

Executive function

Experience-sampling method (ESM)

Facet Theory

Field dependence

Foolishness

Forgiveness

Former leadership
15 General intelligence

222, 232 Goal coordination

198 Goals

222 Happiness

3 Health care systems

69 Ideal image

43 Identity

87 Immigrant

24,96 Interest

154 International

69 Intervention study

32 Invulnerability fallacy

154 Israeli

222, 245 Job learning

232 Loneliness

232 Longitudinal study

78 Managers

180 Memory

193, 210, 232, $245 \quad$ Men

3 Methadone

163 Migrant personality

96 Morality

163 Motivation

32

32

107

145

210

78

264

180

96

210

87

43

145

78

180
Motivational orientation

Multiple methods

Multiple research methodologies

Negative symptoms

Nurses

Omnipotence fallacy

Omniscience fallacy

Orthographic consistency

Parenting

Phonological encoding

Phonological loop

PISA

Practice

Predictive validity

Processing demands
Professional psychological services

Proximal classroom context 198

Psychodiagnostics $\quad 170$

Psychological practice $\quad 180$

Psychology 264

Psychotechnics 170

Reaction time 24

Real-life learning environment 193

Repentance $\quad 78$

Research methods 257

Schizophrenia 96

School reform $\quad 125$

Self-regulated learning $\quad 257$

Self-regulation 245

Slovenian Psychological

Association

180

Smallest Space Analysis $\quad 87$

Social capital $\quad 125$

Social development $\quad 125$

Socio-economics $\quad 107$

Sociocultural 245

Sociocultural models $\quad 257$

Spelling development 3

Student's goals 198

Subjective well-being $\quad 107$

Teachers 69

Trainability tests $\quad 56$

Unemployment 56

Unrealistic optimism fallacy $\quad 145$

Values $\quad 154$

Verbal memory $\quad 96$

Visuospatial sketchpad $\quad 32$

Vocational education $\quad 210$

Wisdom 145

Work and organizational psychology

Working memory 32

Writing 32 